Pacific Journal of Mathematics

ON CAUCHY'S THEOREM FOR REAL ALGEBRAIC CURVES 


\title{
ON CAUCHY'S THEOREM FOR REAL ALGEBRAIC CURVES WITH BOUNDARY
}

\author{
Norman L. ALLING
}

\begin{abstract}
On a real algebraic curve with a nonempty boundary, one must orient the several boundary components in order to pose the question considered in Cauchy's theorem for analytic differentials. It is proved that the conclusion of Cauchy's theorem is true, in this context, if and only if the orientation in question is induced by an orientation of the interior of the curve.
\end{abstract}

Let $\mathfrak{Y}$ be a real algebraic curve (i.e., a compact Klein surface $[3,4])$, whose boundary $\partial Y$ has $r$ components, where $r>0$. Let $g$ be the algebraic genus of $\mathfrak{Y}$ : i.e., the genus of the field $E$ of meromorphic "functions" on $\mathfrak{Y}$ that are real valued on $\partial Y$; then $g$ is the first Betti number of $Y$, the underlying space of $\mathfrak{Y}$, and the Euler characteristic $\chi$ of $Y$ is $1-g[4,2] . \quad Y$ is-of course-characterised topologically by knowing $g, r$ and whether or not $Y$ is orientable. In [2] the author investigated some sheaves that arise from analytic problems on $\mathfrak{Y}$, whose cohomology groups reflect the orientability of $Y$; however these sheaves and groups seemed rather remote from analytic function theory on $\mathfrak{Y}$. This paper is an outgrowth of the search for a simple analytic question which could be posed on $\mathfrak{Y}$, whose answer would reflect the orientability of $Y$. What analytic question on $\mathfrak{Y}$ is, after all, more basic than Cauchy's theorem?

In order to pose the question considered in Cauchy's Theorem on $\mathfrak{Y}$, we must orientate the $r$-components of $\partial Y$; there are $2^{r}$ ways to do this. If $Y$ is orientable, then two of these $2^{r}$ orientations are engendered by the two possible orientations of $Y$; these will be called indigenous orientations of $\partial Y$. If $Y$ is nonorientable, then $\partial Y$ has no indigenous orientations. Let $\mathcal{O}$ be an orientation of $\partial Y$.

Next we must have a space of analytic differentials on $\mathfrak{Y}$ to integrate along $\partial Y$, as orieted by $O$. A space $\Omega_{9}$ of analytic “differentials" on $\mathfrak{Y}$ was defined in [4] which is the natural generalization of the space of Schottky differentials on a bordered Riemann surface, in that they are real on $\partial Y$. (The space of meromorphic differentials on $\mathfrak{Y}[4,1.10]$ is also very natural from the point of view of the algebraic geometry of $E$.) Even though $\omega \in \Omega_{\eta}$ is called a "differential" its integral along an oriented Jordan curve, or arc $\Gamma$, need not have an invariant meaning! If $\Gamma \subset \partial Y$ then it does, and $\int_{\Gamma} \omega \in \boldsymbol{R}$. The real 
part of $\int_{\Gamma} \omega$ always has an invariant meaning. If $\Gamma$ is contained in an orientable tubular neighborhood, then $\int_{\Gamma} \omega$ is always invariantly defined, up to complex conjugation. The real dimension of $\Omega_{\eta}$ is $g$ [2]. (The reason it is so small is that the condition that $\omega$ be real on $\partial Y$ allows it to be extended to the complex double.)

$\lambda_{\odot}: \omega \in \Omega_{\mathfrak{Y}} \mapsto \int_{\partial Y, O} \omega \in \boldsymbol{R}$ is a well defined $\boldsymbol{R}$-linear functional on $\Omega_{\mathrm{g}}$ whose image is either of dimension 0 or 1 . Accordingly we will say that the conclusion of Cauchy's theorem holds on $\mathfrak{Y}$ for $\mathcal{O}$, or does not hold on $\mathfrak{Y}$ for $\mathcal{O}$.

THEOREM 1. The conclusion of Cauchy's theorem holds on $\mathfrak{Y}$ for an orientation $\bigcirc$ of $\partial Y(\neq \varnothing)$ if and only if $\mathcal{O}$ is an indigenous orientation of $\partial Y$ (i.e., one induced by an orientation of $Y$ ). Thus $Y$ is orientable (resp. nonorientable) if and only if there exists 2 (resp. 0 ) orientations $\mathcal{O}$ of $\partial Y$ for which the conclusion of Cauchy's theorem holds on $\mathfrak{Y}$.

Proof. Assume first that $O$ is an indigenous orientation of $\partial Y$; then, by definition, $\mathcal{O}$ is induced by an orientation of $Y$. In the dianalytic structure on $Y$ that gives $\mathfrak{Y}$ we may choose an analytic structure and thus consider the bordered Riemann surface structure $\mathfrak{V}_{1}$ on $Y$ that engenders $O$ on $\partial Y$ [4]. Then $\Omega_{\mathrm{g}}$ becomes the space of Schottky differentials on $\mathfrak{Y}_{1}$. In this context the Cauchy theorem is known to hold. (This can be shown directly by triangulating $Y$ and using the Cauchy theorem in $C$.) Assume, henceforth that $O$ is nonindigenous.

It is well known (see e.g., [6]), that a topological model of $Y$ can be built up from a closed unit disc $D$, by adjoining various strips and handles to it. Adopting a different construction suited to our purposes, first adjoin to $D r-1$ untwisted strips-glued to the boundary of $D$-to form $D^{\prime}$ so that $\partial D^{\prime}$ has $r$ components. Let $D^{\prime}$ be imbedded in $C$ (in some way), let $\Gamma_{1}, \cdots, \Gamma_{r-1}$ be the components of $\partial D^{\prime}$ that bound bounded components of $C-D^{\prime}$, and let them be positively oriented relative to $D^{\prime}$ (as oriented by $C$ ). The Euler characteristic of $D^{\prime}, \chi\left(D^{\prime}\right)$, is $2-r$, which is-necessarily-not smaller than $\chi(Y)$. Next choose the largest integer $h$ such that $2-r-2 h \geqq \chi(Y)$, in the orientable case or $2-r-2 h \geqq \chi(Y)+1$ in the nonorientable. Adjoin $h$ handles to $D^{\prime}$-by removing $2 h$ open discs from $D^{\prime}$, whose boundaries do not meet $\partial D^{\prime}$, and attaching $h$ handles (each on the same side of $D^{\prime}$ ), to some choice of $h$-pairs of these circular boundaries-to form $D_{0}$. If $Y$ is orientable, then $Y$ and $D_{0}$ are homeomorphic. In this event let $Y$ and $D_{0}$ be 
identified. If $Y$ is nonorientable, let $D_{1}$ be formed by adjoining a half twisted strip to $D_{0}$; then $\chi\left(D_{1}\right)=2-r-2 h-1$, which is either $\chi(Y)$ or is $\chi(Y)+1$. In the first case $Y$ and $D_{1}$ are homeomorphic, and are to be identified. In the second let one more half twisted strip be adjoined to $D_{1}$ to form $D_{2}$, a space that is homeomorphic to $Y$; then let $D_{2}$ and $Y$ be identified. In general, $\chi(Y)=2-r-2 h-m$, where $m=0,1$, or 2 , and $Y$ and $D_{m}$ are identified. Let $\Gamma_{r}$ be $\partial Y-\left(\Gamma_{1} \cup \cdots \cup \Gamma_{r-1}\right)$, and let it be oriented in such a way that $\Gamma_{j}$ and $\Gamma_{r}$ have opposing orientations on the untwisted strip adjoined to $D$ to form $D^{\prime}$, which gives rise to $\Gamma_{j}$, for eace $1 \leqq j<r$.

The main analytic technique we will use, that of doubling $\mathfrak{Y}$, goes back-essentially to Schottky and Schwarz-and explicitly, in this context, to Klein. Let $(\mathfrak{X}, \tau, p)$ be the complex double of $\mathfrak{Y}[4$, 1.6]: i.e., $\mathfrak{X}$ is a compact Riemann surface (without boundary), $\tau$ is an anti-analytic involution of $\mathfrak{X}$, and $p$ is an analytic map of $\mathfrak{X}$ onto Y (i.e., a morphism $[4,1.4]) . p^{-1}(y)$ has one (resp. two) points in it, for $y \in Y$, if and only if $y \in \partial Y$ (resp. $y \in Y-\partial Y$ ). For $1 \leqq j \leqq r$, let $\Delta_{j}$ be the pullback of $\Gamma_{j}$ to $X$, endowed with the orientation induced on it by the orientation of $\Gamma_{j}$; thus $\Delta_{j}$ is an oriented Jordan curve in $X$. For $1 \leqq j<r$, let $a_{j} \equiv \Delta_{j}$. It is easy to see that these, regarded as elements in $H_{1}(X, Z)$, are part of the usual $a$-paths (see e.g., [7, Chapt. 10] for details), which arise from doubling the $r-1$ untwisted strips that were adjoined to $D$ to form $D^{\prime}$. $\tau$ induces an involution $\sigma$ on $\Omega_{x}$, the $C$-space of analytic differentials on $\mathfrak{X}$, which is $R$-linear, such that $\sigma(i \omega)=-i \sigma(\omega)$, for each $\omega \in \Omega_{\mathfrak{x}} . \quad \Omega_{\mathfrak{x}}$ is then the direct sum of the $R$-space, $\Omega_{\mathfrak{x}, s}$, of symmetric elements of $\Omega_{\mathfrak{x}}$, and the $R$-space $\Omega_{\mathfrak{x}, a}$ of anti-symmetric elements of $\Omega_{x}$. Further $\Omega_{\mathfrak{x}, a}=i \Omega_{x, s}$; thus the real dimensions of $\Omega_{x, a}$ and $\Omega_{x, s}$ are the same, namely $g$, the genus of $\mathfrak{X}$-which is also the algebraic genus of $\mathfrak{Y}$. Given $\omega \in \Omega_{x}$, let $\omega=\rho+\zeta$ where $\rho$ is symmetric and $\zeta$ is anti-symmetric. This convention will hold throughout the paper. Further $\Omega_{\mathfrak{g}}$ can be naturally identified with $\Omega_{\mathfrak{x}, s}$ (see [4], [1], and [2] for more details).

Lemma. Let $a$ be an oriented Jordan curve (arc) in $X$ such that $\tau(a)=a$, and assume that $\int_{a} \omega \equiv t$ is real; then $\int_{a} \rho=t$ and $\int_{a} \zeta=0$.

Proof. Using [1, 3.1], we see that $\int_{a} \rho=\kappa \circ \int_{\tau(a)} \rho=\kappa \circ \int_{a} p$, so $\int_{a} \rho \in \boldsymbol{R} . \quad \zeta=i \eta$ for some $\eta \in \Omega_{x, s}$, so $\int_{a} \zeta=i \int_{a} \eta \in i \boldsymbol{R}$, proving the lemma.

Returning now to the proof of Theorem 1, first let us treat the (trivial) case in which $Y$ is orientable, or equivalently in which $m=$ 
0 . Then $Y$ and $D_{0}$ are identified, and $\Gamma_{1}+\cdots+\Gamma_{r}=0$ in $H_{1}(Y, Z)$. The orientation $\mathcal{O}$, of $\partial Y$, can be given by choosing $e_{j} \in\{ \pm 1\}, 1 \leqq$ $j \leqq r$, and by assigning each $e_{j}$ to $\Gamma_{j}$. Since $\mathcal{O}$ is nonindigenous, not all the $e_{j}$ 's are alike. $\left\{a_{1}, \cdots, a_{r-1}\right\}$ is contained in a basis $B_{0} \equiv$ $\left\{a_{1}, b_{1}, \cdots, a_{g}, b_{g}\right\}$ of $H_{1}(X, Z)$ such that

a)

$$
a_{1} \cdot b_{1} \cdot a_{1}^{-1} \cdot b_{1}^{-1} \cdots \cdots \cdot a_{g} \cdot b_{g} \cdot a_{g}^{-1} \cdot b_{g}^{-1}=1 \text {, in } \pi_{1}(X) \text {. }
$$

Let $\left\{\omega_{1}, \cdots, \omega_{g}\right\}$ be a basis of $\Omega_{x}$ over $C$ such that $\int_{a_{j}} \omega_{k}=\delta_{j_{k}}, 1 \leqq$ $j, k \leqq g$. (See e.g., [7, Chapt. 10] for details.) As noted above there exists $k, 1 \leqq k<r$, such that $e_{k} \neq e_{r}$. Using, among other things, the lemma, we find that

$$
\int_{\partial Y, \sigma} \rho_{k}=\sum_{j=1}^{r} e_{j} \int_{\Delta_{j}} \rho_{k}=e_{k} \int_{\Delta_{k}} \rho_{k}+e_{r} \int_{\Delta_{r}} \rho_{k} .
$$

Since $\Gamma_{1}+\cdots+\Gamma_{r}=0$ in $H_{1}(Y, Z), \Delta_{r}=-\Delta_{1}-\cdots-\Delta_{r-1}$ in $H_{1}(X$, $Z)$; thus $e_{r} \int_{\Delta_{r}} \rho_{k}=-e_{r} \int_{\Delta_{k}} \rho_{k}$, by Cauchy's theorem, and thus is $-e_{r}$, and so $\int_{\partial Y, O} \rho_{k}=e_{k}-e_{r} \not 0$, disposing of the proof, if $Y$ is orientable. Before going on to the nonorientable cases, let us consider a useful example.

ExAmple. Let $\alpha \equiv 1 / 2+b i$, where $b>0$, and consider $G \equiv Z \bigoplus$ $\alpha Z$ in $C$. Let $G$ act as a set of conformal maps of $C$, by translation. Note that $G$ is invariant under $\kappa$, where $\kappa$ is complex conjugation. Let $\mathfrak{X} \equiv C / G$, and let $\tau$ be the anti-analytic involution on $\mathfrak{X}$ induced by $\kappa$. The parallelogram whose vertices are $0, \bar{\alpha}, 1$, and $\alpha$ can be taken as a fundamental domain for $\mathfrak{X}$. The interval $[0,1]$ is the set of fixed points of this domain under the action of $\kappa$. Let $\mathfrak{Y} \equiv$ $\mathfrak{X} /\{1, \tau\}$; then $\mathfrak{Y}$ is a Möbius strip, the image of $[0,1]$ in $Y$ being its boundary. The isosceles triangle, whose vertices are 0,1 and $\alpha$, may be taken as the fundamental domain for $\mathfrak{Y}$. Given $\beta$ in the straight line segment $[0, \alpha]$, it will be identified-when passing to $\mathfrak{Y}$-with $\bar{\beta}+\alpha$ in $[\alpha, 1] . d z$ induces a basis $\{d z\}$ of $\Omega_{\mathfrak{y}}$ over $\boldsymbol{R}$. Since $\int_{0}^{1} d z=$ 1 , we see that the conclusion of Cauchy's theorem is never true for $\mathfrak{~ Y . ~ ( I t ~ i s ~ a l s o ~ n o t ~ h a r d ~ t o ~ s h o w ~ t h a t ~ a l l ~ d i a n a l y t i c ~ M o ̈ b i u s ~ s t r i p s ~}$ occur in this way.)

Returning again to the proof of Theorem 1, note that the triangle, described in the above example and identified as indicated, is a Möbius strip. Assume now that $Y$ is nonorientable: i.e., $m>0$. We can modify the construction of $D_{m}$ from $D_{0}$ as follows. Let one end of a strip be glued to $[1 / 3,2 / 3]$ in the Möbius strip above. The resulting space will be referred to as a Christmas tree. Glue the other end of the strip that forms the trunk of the Christmas tree to the edge 
of $D_{0}$ to form $D_{1}$. Repeat the procedure on $D_{1}$ to form $D_{2}$. Let $A_{r}$ and $\Lambda_{r+m-1}$ be the boundaries of the Möbius strip-in the form given in the example-before the trunk is glued on, and then glued to $D_{0}$, and $D_{m-1}$. Let them be oriented to agree with the orientation of $\Gamma_{r}$. In doubling $\mathfrak{Y}$ to form $\mathfrak{X}$, the Christmas tree doubles to a torus with a tube running from a hole in it, back to the rest of $X$. $\Lambda_{j}$ lifts to a (nonunique) path $\Delta_{j}$ in $X, r \leqq j \leqq r+m-1$. Let these paths be oriented by the paths onto which they map. Let $a_{r} \equiv \Delta_{r}$ and let $a_{r+m-1} \equiv \Delta_{r+m-1} . \quad\left\{a_{1}, \cdots, a_{r}, a_{r+m-1}\right\}$ is contained in a basis $B_{m} \equiv\left\{a_{1}, b_{1}, \cdots, a_{g}, b_{g}\right\}$ of $H_{1}(X, Z)$ that satisfies condition a) above. Thus there exists a basis $\left\{\omega_{1}, \cdots, \omega_{g}\right\}$ of $\Omega_{x}$ over $C$ such that $\int_{a_{j}} \omega_{k}=\delta_{j_{k}}$, $1 \leqq j, k \leqq g$. (See e.g., [7, Chapt. 10] again.) Let $\rho_{k}$ be the symmetric component of $\omega_{k}$, and let $\rho \equiv \sum_{k=1}^{m} \rho_{r+k-1}$; then $\rho \in \Omega_{\mathfrak{y}}$. There exist $e_{j} \in\{ \pm 1\}, 1 \leqq j \leqq r$, such that $\int_{\partial Y, O} \rho=\sum_{j=1}^{r} e_{j} \int_{\Gamma_{j}} \rho$. Since-for $1 \leqq j<$ $r-\tau\left(a_{j}\right)=a_{j}$, and since $\int_{a_{j}} \omega_{r+k-1}=0, k=1$ or 2 , for such $j$ 's, we may apply the lemma and conclude that $\int_{a_{j}} \rho=0$; thus $\sum_{j=1}^{r} e_{j} \int_{\Gamma_{j}} \rho=$ $e_{r} \int_{\Gamma_{r}} \rho$. Assume now that $m=1$. Since we may invoke Cauchy's theorem on an orientable sub-domain of $\mathfrak{Y}$, we find that $\int_{\Gamma_{r}} \rho= \pm$ $\int_{a_{r}} \rho$. Let $a_{r}$ be re-oriented so that positive sign above holds. Since $\int_{a_{r}}^{a_{r}} \omega_{r}=1$, we may reason as we did above. Since $\int_{\Gamma_{r}} \omega_{r}=\int_{a_{r}} \omega_{r}=1$, and since $\tau\left(\Gamma_{r}\right)=\Gamma_{r}$, we may apply the lemma and conclude that $\int_{r_{r}} \rho=1$. Thus $\int_{\partial Y, o} \rho=e_{r} \neq 0$. Assume lastly that $m=2$. Reasoning as above $\int_{\Gamma_{r}} \omega_{r}+\omega_{r+1}=\int_{ \pm a_{r} \pm a_{r+1}} \omega_{r}+\omega_{r+1}$, the signs being independent, one of the other. If necessary, re-orient $a_{r}$ or $a_{r+1}$ so that the plus sign holds twice above; thus $\int_{r_{r}} \omega_{r}+\omega_{r+1}=2$. Since $\tau\left(\Gamma_{r}\right)=\Gamma_{r}$, we may apply the lemma and conclude that $\int_{\partial Y, O} \rho=e_{r} \int_{\Gamma_{r}} \rho=2 e_{r} \neq 0$, proving the theorem.

Greenleaf and Read considered a related question in [5]. Given an orientation $O$ of $\partial Y$ they defined the notion of an analytic differential $\rho$ as being positive at $y$ (in $\partial Y$ ), relative to $\varnothing$ as follows: if given $f \in E$ - a local uniformizer at $y$-that is increasing near $y$, relative to $\mathcal{O}$ then $0<h(y)$, where $\rho=h d f$ for a (unique) $h \in E$. (This definition is independent of the choice of $f$, as may easily be seen.) $\rho$ is said to be positive relative to $\varnothing$ if it is positive at $y$, relative to $\mathcal{O}$, for each $y \in \partial Y$. Greenleaf and Read proved that if $\mathcal{O}$ is indigenous, then no positive analytic differentials exist in $\Omega_{y}$, that if $\mathscr{O}$ is non-indigenous and $\mathfrak{Y}$ is elliptic or hyperelliptic then positive analytic differentials always exist; and then they went on to conjecture that the condition that $\mathfrak{Y}$ be elliptic or hyperelliptic can 
be dropped while preserving the conclusion above. If the GreenleafRead conjecture is correct, then it would imply our theorem; thus our theorem may lend additional credence to their conjecture.

Having set up this machinery, let us use it to draw some additional conclusions. As noted before, the real dimension of $\Omega_{\eta}$ is $g$ and $g=r-1+m+2 h$. Given $\rho \in \Omega_{\mathfrak{y}}$, let

$$
\varphi_{1}(\rho) \equiv \int_{\Gamma_{1}} \rho, \cdots, \varphi_{r-1}(\rho) \equiv \int_{\Gamma_{r-1}} \rho
$$

Clearly these maps are real valued. If $m \geqq 1$, let $\varphi_{r}(\rho) \equiv \int_{\Delta_{r}} \rho$. If $m=2$, let $\varphi_{r+1}(\rho) \equiv \int_{\Delta_{r+1}} \rho$. Let $r \leqq k \leqq r+m-1$. By [1, 3.1], $\int_{\tau\left(A_{k}\right)} \rho=\kappa\left(\phi_{k}(\rho)\right)$. Since $\Delta_{k}$ and $\tau\left(\Delta_{k}\right)$ are homologous, $\int_{\tau\left(\Delta_{k}\right)} \rho=\phi_{k}(\rho)$, proving that $\phi_{k}(\rho)$ is real. $\phi_{k}(\rho)$ can also be computed by integrating allong $\Lambda_{k}$. Note that $\Lambda_{k}$ lifts to $\Delta_{k}$ and to $\tau\left(\Delta_{k}\right)$, and that the integral of $\rho$ allong each of these paths is the same. Let $\Theta_{j}$ be an oriented $a$-path about the $j^{\text {th }}$ handle adjoined to $D^{\prime}$ to form $D_{0}$, for $1 \leqq j \leqq h$.

For each such path we may choose an analytic structure in a tubular neighborhood; then integration of $\rho$ along each such is well defined. As remarked before the real parts of these integrals are a priori well defined, whereas the imaginary parts are a priori well defined only up to sign. If $h>0$, let $\varphi_{g}(\rho) \equiv \operatorname{Re} \int_{\theta_{1}} \rho, \varphi_{g-1}(\rho) \equiv \pm$ $\operatorname{Im} \int_{\theta_{1}} \rho, \cdots, \varphi_{g-2 h+2}(\rho) \equiv \operatorname{Re} \int_{\theta_{h}} \rho$, and $\varphi_{g-2 h+1}(\rho) \equiv \pm \operatorname{Im} \int_{\theta_{h}} \rho$, the signs above being independent of one another.

THEOREM 2. Given $\rho \in \Omega_{y}, \rho=0$ if and only if $\varphi_{1}(\rho)=\cdots=$ $\varphi_{g}(\rho)=0$.

Proof. Since each of the $\phi_{j}^{\prime}$ 's is an $R$-linear functional, $\rho=0$ implies that each $\varphi_{j}(\rho)=0$. Conversely, let $\rho \in \Omega_{y}$ such that $\varphi_{j}(\rho)=$ 0 , for each $j, 1 \leqq j \leqq g$. As noted in the proof of Theorem $1, \Gamma_{j}$ lifts to $a_{j} \in H_{1}(X, Z)$ for $1 \leqq j \leqq r-1$. If $m>0, \Lambda_{r}$ lifts to $a_{r}$, and if $m=2, \Lambda_{r+1}$ lifts to $a_{r+1}$. For $1 \leqq j \leqq h, \Theta_{j}$ lifts to two oriented $a$-paths on $X, a_{g-2 j+2}$ and $a_{g-2 j+1}$, which are permuted by $\tau$. Finally, $\left\{a_{1}, \cdots, a_{g}\right\}$ is contained in a basis $B \equiv\left\{a_{1}, b_{1}, \cdots, a_{g}, b_{g}\right\}$ of $H_{1}(X, Z)$ such that condition a) holds. By $[1,3.1]$, the integral of $\rho$ about $a_{g-2 j+2}$ and about $a_{g-2 j+1}$ are complex conjugates of each other. Because of this symmetry, to know that $\rho$ is zero it suffices to know that its periods with respect to $a_{1}, \cdots, a_{r-1}, \cdots, a_{r-1+m}, a_{g}, a_{g-2}, \cdots, a_{g-2 h+4}$, and $a_{g-2 h+2}$ are all zero: i.e., the periods of $\rho$ with respect to $\Gamma_{1}, \cdots, \Gamma_{r-1}, \cdots, \Lambda_{r-1+m}, \Theta_{1}, \Theta_{2}, \cdots, \Theta_{h-1} \Theta_{h}$ are all zero; but this is implied by the condition that $\varphi_{1}(\rho)=\cdots=\varphi_{g}(\rho)=0$, proving the theorem. 
CoRollaRy. There exists a unique basis $\left\{\rho_{1}, \cdots, \rho_{g}\right\}$ in $\Omega_{\eta}$ of $\Omega_{\eta}$ over $\boldsymbol{R}$ (resp. in $\Omega_{\mathfrak{g}}$ of $\Omega_{x}$ over $\boldsymbol{C}$ ), such that $\varphi_{j}\left(\rho_{j}\right)=\delta_{j_{k}}$, for all $1 \leqq$ $j, k \leqq g$.

Bibliographic note. See also $[8,9]$ for related results on the period matrix of a symmetric Riemann surface.

\section{REFERENCES}

1. N. L. Alling, Analytic and harmonic obstruction on nonorientable Klein surfaces, Pacific J. Math., 36 (1971), 1-19.

2. - Analytic qeometry on real algebraic curves, Math. Ann., 207 (1974), 23-46. 3. N. L. Alling and N. Greenleaf, Klein surfaces and real algebraic function fields, Bull. Amer. Math. Soc., 75 (1969), 869-872.

4. - Foundations of the Theory of Klein Surfaces and Real Algebraic Function Fields, Springer-Verlag Lecture Notes in Mathematics, vol. 219 (1971).

5. N. Greenleaf and W. Read, Position holomorphic differentials on Klein surfaces, Pacific J. Math., 32 (1970), 711-713.

6. W. S. Massey, Algebraic Topology: An Introduction, Harcourt, Brace and World, 1967.

7. G. Springer, Introduction to Riemann Surfaces, Addison-Wesley, 1957.

8. G. Weichold, Über symmetrische Riemannsche Flächen und die Periodizitätsmoduln der zugchörigen Abelschen Normalintegrale erster Gettung, Zeitschrift f. Math. u. Phys., 28 (1883), 321-351.

9. R. Zarrow, Symmetric and quasi-symmetric Riemann surfaces, unpublished dissertation, Cornell University, 1972.

Received November 16, 1973. The author wishes to thank Walter Read, the Referee, for his careful reading and many constructive criticisms which have been incorporated in this paper.

The UnIVersity of ROchester 



\section{PACIFIC JOURNAL OF MATHEMATICS}

\section{EDITORS}

RICHARD ARENS (Managing Editor)

University of California

Los Angeles, California 90024

\section{J. DugundJI}

Department of Mathematics University of Southern California Los Angeles, California 90007

D. Gilbarg and J. Milgram

Stanford University

Stanford, California 94305
University of Washington Seattle, Washington 98105

\section{ASSOCIATE EDITORS}
E. F. BECKENBACH
B. H. NeumanN
F. WolF
K. YoShIDA

\section{SUPPORTING INSTITUTIONS}

\author{
UNIVERSITY OF SOUTHERN CALIFORNIA \\ STANFORD UNIVERSITY \\ UNIVERSITY OF TOKYO \\ UNIVERSITY OF UTAH \\ WASHINGTON STATE UNIVERSITY \\ UNIVERSITY OF WASHINGTON \\ $\stackrel{*}{*} \stackrel{*}{*} \stackrel{*}{ }{ }^{*}$ AMERICAN MATHEMATICAL SOCIETY
}

The Supporting Institutions listed above contribute to the cost of publication of this Journal, but they are not owners or publishers and have no responsibility for its content or policies.

Mathematical papers intended for publication in the Pacific Journal of Mathematics should be in typed form or offset-reproduced, (not dittoed), double spaced with large margins. Underline Greek letters in red, German in green, and script in blue. The first paragraph or two must be capable of being used separately as a synopsis of the entire paper. Items of the bibliography should not be cited there unless absolutely necessary, in which case they must be identified by author and Journal, rather than by item number. Manuscripts, in triplicate, may be sent to any one of the editors. Please classify according to the scheme of Math. Reviews, Index to Vol. 39. All other communications should be addressed to the managing editor, or Elaine Barth, University of California, Los Angeles, California, 90024.

The Pacific Journal of Mathematics expects the author's institution to pay page charges, and reserves the right to delay publication for nonpayment of charges in case of financial emergency.

100 reprints are provided free for each article, only if page charges have been substantially paid. Additional copies may be obtained at cost in multiples of 50 .

The Pacific Journal of Mathematics is issued monthly as of January 1966. Regular subscription rate: $\$ 72.00$ a year (6 Vols., 12 issues). Special rate: $\$ 36.00$ a year to individual members of supporting institutions.

Subscriptions, orders for back numbers, and changes of address should be sent to Pacific Journal of Mathematics, 103 Highland Boulevard, Berkeley, California, 94708.

\section{PUBLISHED BY PACIFIC JOURNAL OF MATHEMATICS, A NON-PROFIT CORPORATION}

Printed at Kokusai Bunken Insatsusha (International Academic Printing Co., Ltd.), 270, 3-chome Totsuka-cho, Shinjuku-ku, Tokyo 160, Japan.

\section{Copyright (C) 1975 by Pacific Journal of Mathematics} Manufactured and first issued in Japan 


\section{Pacific Journal of Mathematics}

\section{Vol. 57, No. $2 \quad$ February, 1975}

Norman Larrabee Alling, On Cauchy's theorem for real algebraic curves with boundary .......

Daniel D. Anderson, A remark on the lattice of ideals of a Prüfer domain ..................

Dennis Neal Barr and Peter D. Miletta, A necessary and sufficient condition for uniqueness of

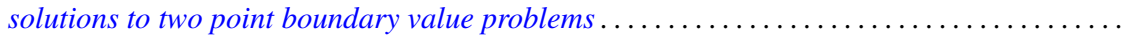

Ladislav Beran, On solvability of generalized orthomodular lattices . . . . . . . . . . ........

L. Carlitz, A three-term relation for some sums related to Dedekind sums . . . . . . . . . .....

Arthur Herbert Copeland, Jr. and Albert Oscar Shar, Images and pre-images of localization

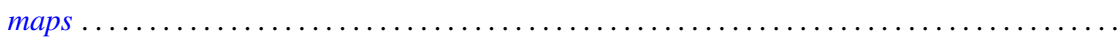

G. G. Dandapat, John L. Hunsucker and Carl Pomerance, Some new results on odd perfect

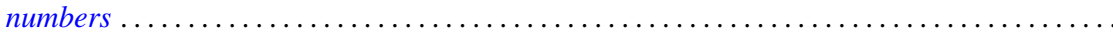

M. Edelstein and L. Keener, Characterizations of infinite-dimensional and nonreflexive

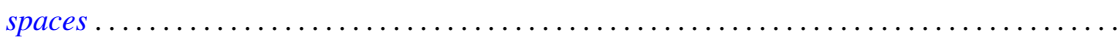

Francis James Flanigan, On Levi factors of derivation algebras and the radical embedding

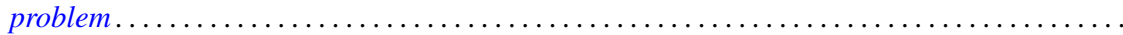

Harvey Friedman, Provable equality in primitive recursive arithmetic with and without

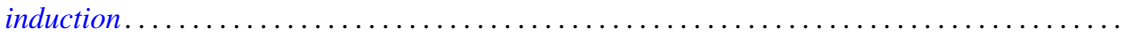

Joseph Braucher Fugate and Lee K. Mohler, The fixed point property for tree-like continua with

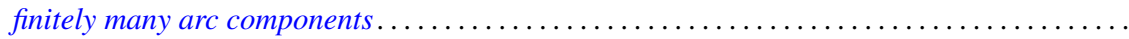

John Norman Ginsburg and Victor Harold Saks, Some applications of ultrafilters in

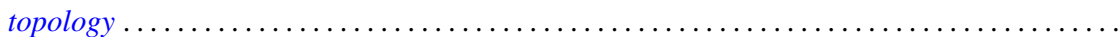

Arjun K. Gupta, Generalisation of a "square" functional equation .....................

Thomas Lee Hayden and Frank Jones Massey, Nonlinear holomorphic semigroups ..........

V. Kannan and Thekkedath Thrivikraman, Lattices of Hausdorff compactifications of a locally

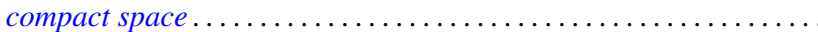

J. E. Kerlin and Wilfred Dennis Pepe, Norm decreasing homomorphisms between group

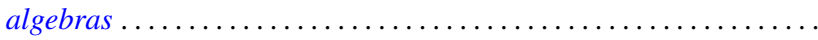

Young K. Kwon, Behavior of $\Phi$-bounded harmonic functions at the Wiener boundary ...

Richard Arthur Levaro, Projective quasi-coherent sheaves of modules .

Chung Lin, Rearranging Fourier transforms on groups...........................

David Lowell Lovelady, An asymptotic analysis of an odd order linear differential equation . . 4475

Jerry Malzan, On groups with a single involution .......................... 481

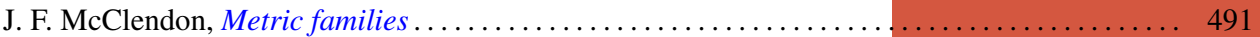

Carl Pomerance, On multiply perfect numbers with a special property .

Mohan S. Putcha and Adil Mohamed Yaqub, Polynomial constraints for finiteness of semisimple rings. .

Calvin R. Putnam, Hyponormal contractions and strong power convergence . . . . . . . . . 531

Douglas Conner Ravenel, Multiplicative operations in $\mathrm{BP} * \mathrm{BP} \ldots \ldots \ldots \ldots \ldots \ldots \ldots \ldots \ldots .539$

Judith Roitman, Attaining the spread at cardinals which are not strong limits . . . . . . . . . 545

Kazuyuki Saitô, Groups of *-automorphisms and invariant maps of von Neumann algebras . . . 553

Brian Kirkwood Schmidt, Homotopy invariance of contravariant functors acting on smooth

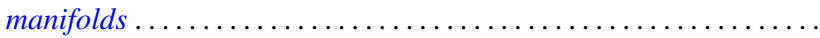

Kenneth Barry Stolarsky, The sum of the distances to $N$ points on a sphere.

Mark Lawrence Teply, Semiprime rings with the singular splitting property.

J. Pelham Thomas, Maximal connected Hausdorff spaces..............

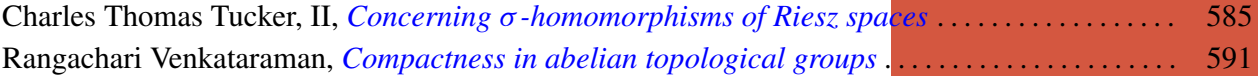

William Charles Waterhouse, Basically bounded functors and flat sheaves . . . . . . . . . . . 597

David Westreich, Bifurcation of operator equations with unbounded linearized part ......... 611

William Robin Zame, Extendibility, boundedness and sequential convergence in spaces of 